\title{
CONHECIMENTO DOS PROFESSORES SOBRE O MANEJO DA CRIANÇA COM DIABETES MELLITUS ${ }^{1}$
}

\author{
Ana Lúcia de Assis Simões², Thaís Santos Guerra Stacciarin ${ }^{3}$, Márcia Tasso Dal Poggetto ${ }^{4}$, Harriet Bárbara \\ Maruxo ${ }^{5}$, Helga Marízia Soares ${ }^{6}$, Ana Carolina de Assis Simões ${ }^{7}$
}

\footnotetext{
${ }^{1}$ Trabalho desenvolvido com apoio financeiro da Fundação de Amparo à Pesquisa do Estado de Minas Gerais

${ }^{2}$ Doutora em Enfermagem. Professora associado do Curso de Graduação em Enfermagem da Universidade Federal do Triângulo Mineiro (UFTM). Minas Gerais, Brasil. E-mail: assisimoes@yahoo.com.br

${ }^{3}$ Doutoranda pelo Programa de Enfermagem Fundamental da Escola de Enfermagem de Ribeirão Preto da Universidade de São Paulo. Chefe do Serviço de Educação em Enfermagem do Hospital de Clínicas da UFTM. Minas Gerais, Brasil. E-mail: thais.stacciarini@terra.com.br

${ }^{4}$ Mestre em Enfermagem. Professora assistente do Curso de Graduação em Enfermagem da UFTM. Minas Gerais, Brasil. E-mail: marciatasso60@yahoo.com.br

${ }^{5}$ Enfermeira. Residente em Cardiologia pelo Instituto Dante Pazzanese. São Paulo, Brasil. E-mail: harri_babi@hotmail.com

${ }^{6}$ Acadêmica do $8^{\circ}$ período do Curso de Graduação em Enfermagem da UFTM. Minas Gerais, Brasil. E-mail: helguinhamarizia@ yahoo.com.br

${ }^{7}$ Dentista. Mestranda do Programa de Atenção à Saúde da UFTM. Minas Gerais, Brasil. E-mail: aninhassim@yahoo.com.br
}

RESUMO: Trata-se de um estudo seccional e descritivo que objetivou verificar o conhecimento dos professores sobre o diabetes mellitus e as dificuldades encontradas no manejo da criança com diabetes. Participaram 184 professores da educação infantil de escolas municipais do município de Uberaba-MG, entre os meses de abril e julho de 2009. Os dados foram coletados por meio de questionário e analisados por estatística descritiva. Os resultados evidenciaram que os professores investigados denotaram conhecimento sobre o que é diabetes (58,15\%); no entanto, demonstraram desconhecer as manifestações clínicas (27,72\%), as abordagens terapêuticas (33,70\%) e as principais condutas adotadas diante de situações adversas (42,40\%). Este estudo contribuiu para enfatizar a necessidade de capacitar os professores da educação infantil na temática diabetes mellitus, a fim de possibilitar a abordagem à criança com conhecimento e segurança.

DESCRITORES: Enfermagem. Diabetes mellitus. Saúde da criança. Educação em saúde.

\section{KNOWLEDGE OF THE TEACHERS ABOUT THE MANAGEMENT OF THE DIABETIC CHILDREN}

\begin{abstract}
This is a descriptive and sectional study that aimed to verify the teachers' knowledge about the diabetes mellitus and the difficulties found in the management of children with diabetes. Participated 184 teachers of infant education in municipal schools of the city of Uberaba, Minas Gerais, between the months of April to July 2009. Data were collected through questionnaire and analyzed using descriptive statistics. The results showed that investigates teachers had denoted knowledge about what is diabetes (58.15\%), however, showed disregard the clinical manifestations $(27.72 \%)$, therapeutic approaches $(33.70 \%)$ and the main procedures to be adopted in adverse situations $(42.40 \%)$. This study contributed to emphasize the need to train teachers of the infant education in thematic diabetes in the subject, to enable the approach to children with knowledge and safety.
\end{abstract}

DESCRIPTORS: Nursing. Diabetes mellitus. Child health. Health education.

\section{EL CONOCIMIENTO DE LOS PROFESORES SOBRE EL MANEJO DE LOS NIÑOS DIABÉTICOS}

RESUMEN: Este estudio descriptivo de corte transversal tuvo como objetivo verificar el conocimiento sobre la diabetes mellitus y las dificultades encontradas por los profesores en el manejo de los niños diabéticos. El estudio se hizo con 184 profesores de educación preescolar en las escuelas municipales del municipio de Uberaba-MG, entre abril y julio de 2009. Los datos fueron recolectados a través de cuestionario y se analizaron mediante estadística descriptiva. Los resultados mostraron que los profesores entrevistados tienen conocimiento acerca de la diabetes $(58,15 \%)$, sin embargo, mostraron desconocimiento sobre las manifestaciones clínicas $(27,72 \%)$, los enfoques terapéuticos (33,70\%) y los principales procedimientos que se adoptarán en situaciones adversas (42,40\%). Este estudio contribuyó a resaltar la necesidad de capacitar a los maestros sobre el tema de la diabetes mellitus de la primera infancia, para permitir el acercamiento al niño con conocimiento y seguridad.

DESCRIPTORES: Enfermería. Diabete mellitus. Salud del niño. Education en salud. 


\section{INTRODUÇÃO}

Os atuais hábitos de vida da população mundial têm contribuído para o aumento significativo da incidência de doenças crônicas entre os mais jovens. Acredita-se que em $2020,80 \%$ das enfermidades nos países em desenvolvimento serão decorrentes desses distúrbios. ${ }^{1-2}$

As doenças crônicas, alterações de saúde que permanecem por longo período de tempo, causam mudanças no estilo de vida da pessoa que passa a necessitar de acompanhamento e tratamento constantes, ${ }^{3-4}$ principalmente na infância que constitui um período da vida do ser humano caracterizado por intenso crescimento e desenvolvimento, abrangendo aspectos biológicos, psíquicos, emocionais, culturais e sociais. ${ }^{3-6}$

Nesse cenário, o diabetes mellitus tipo 1 (DM1) aparece, mundialmente, como uma das principais doenças crônicas da infância. De acordo com dados norte-americanos, são diagnosticados 11 mil casos por ano ${ }^{5}$ e, no Brasil, algumas estatísticas mostram que dos cinco milhões de pessoas com diabetes, aproximadamente 300 mil têm menos de 15 anos de idade..$^{3-4}$ Pela sua elevada incidência, conhecer o DM1 e saber identificar suas principais manifestações clínicas é imprescindível para o correto diagnóstico e a adequada intervenção.

Para alcançar os objetivos do tratamento e do controle metabólico, mudanças na dinâmica familiar são inevitáveis, pois a criança terá de mudar seus hábitos alimentares, receberá múltiplas aplicações subcutâneas de insulina exógena, dependendo do tipo de DM, e realizará monitorizações glicêmicas frequentes, além das atividades físicas monitoradas. ${ }^{5-9}$

O impacto da doença na vida social da criança e da família também acontece quando diminuem os convites para eventos sociais, como por exemplo: festas de aniversário e Páscoa. Quando a criança passa a ter dificuldades para o ingresso em determinadas escolas, de ser liberada pelo professor para ir ao banheiro com mais frequência durante as aulas ou quando são orientadas a não praticar atividades físicas. Tais fatos acabam excluindo a criança do convívio social e limitando suas possibilidades na teia de inter-relações com outros seres humanos. ${ }^{10}$

Outros aspectos referem-se à preocupação dos pais sobre a atuação dos professores frente ao reconhecimento dos sinais e sintomas de hipoglicemia apresentados por seus filhos, pois acreditam que esses profissionais têm pouca ou nenhuma experiência em lidar com essa situação, ${ }^{11}$ e sobre a possibilidade de discriminação dentro da própria instituição, prejudicando o aprendizado da criança. ${ }^{3}$

A partir dessas considerações, emergem os seguintes questionamentos: o que sabem os professores sobre DM e como lidam com a criança com diabetes no contexto escolar? Levando-se em conta uma questão fundamental do tratamento do DM, a educação, percebe-se a real necessidade de um conjunto de medidas que tem por objetivo aumentar a motivação, a participação e o aprendizado sobre a doença, tanto com o intuito de melhorar a qualidade de vida das crianças com DM, quanto no sentido de orientar a população em relação às medidas de prevenção e de detecção precoce. ${ }^{12}$

Assim, tendo o professor o importante papel de reconhecer e atuar em situações adversas que versam sobre a saúde do escolar, torna-se imprescindível que estejam capacitados. ${ }^{4-5} \mathrm{Nessa}$ perspectiva, observando-se a importância da temática, este estudo foi realizado com o objetivo de verificar o conhecimento dos professores sobre o $\mathrm{DM}$ e as dificuldades encontradas no manejo da criança com diabetes.

\section{POPULAÇÃO E MÉTODO}

Trata-se de um estudo seccional, descritivo e com abordagem quantitativa realizado com professores vinculados às instituições públicas municipais, do município de Uberaba, no Estado de Minas Gerais, importante polo econômico, referência regional nas áreas de saúde e de educação.

Para o acesso aos professores, inicialmente foi feito contato com a Secretaria Municipal de Educação (SME) do município, para esclarecimento dos objetivos da pesquisa, que autorizou a coleta dos dados durante o desenvolvimento da capacitação promovida pelo Departamento de Educação Continuada da SME, com professores da educação infantil.

Assim, a população do estudo constituiuse de todos os professores inscritos no referido programa de capacitação, e que concordaram em participar da pesquisa. Os entrevistados assinaram o Termo de Consentimento Livre e Esclarecido, permitindo a coleta de dados e o uso de suas informações. O projeto foi aprovado pelo Comitê de Ética em Pesquisa com Seres Humanos da Universidade Federal do Triângulo Mineiro, com o protocolo $\mathrm{n}^{\circ} 1004$. Os dados foram coletados entre 
os meses de abril e julho de 2009, por meio da utilização de questionários, entregues pessoalmente aos participantes durante a capacitação.

Trata-se de um instrumento dividido em quatro partes, contemplando: caracterização socioeconômica dos participantes (sexo, idade, situação conjugal, formação, tempo de magistério, vínculo empregatício, turno de trabalho, remuneração); abordagem do conhecimento dos professores sobre DM1 (conceito; sinais e sintomas; incidência; tratamento; dieta); manejo dos educadores com a criança com DM (conhecimento sobre a existência de aluno com diagnóstico médico de DM na escola; contato com os responsáveis pela criança; condutas frente às necessidades da criança; condutas frente ao aluno diabético com hipoglicemia; opinião sobre a prática de atividade física pelas crianças diabéticas; aspectos relacionados à socialização e ao aprendizado da criança) e manejo do diabetes pela direção da escola (conhecimento sobre a existência de criança com diagnóstico médico de DM na escola; comunicação e orientação aos professores; alimentação oferecida pelo refeitório/ lanchonete).

Esse instrumento foi submetido à apreciação de três especialistas em DM, para avaliação da forma de apresentação do conteúdo e da compreensão da linguagem. Para as adequações finais, o instrumento foi aplicado a dez professores do ensino fundamental, não participantes do estudo.

Todos os dados dos questionários foram codificados, categorizados e digitados em um banco de dados no Programa Microsoft Excel, transportados e processados no programa estatístico Epi-info. Dependendo da natureza das variáveis, foram apresentados os dados por meio de análise descritiva (frequência absoluta ou percentual simples) e de dispersão.

A elaboração desta pesquisa resultou do desenvolvimento de um projeto de pesquisa vinculado à atividade de extensão universitária, com apoio financeiro recebido da Fundação de Amparo à Pesquisa do Estado de Minas Gerais.

\section{RESULTADOS}

Dentre os 250 professores inscritos no programa de capacitação promovido pelo Departamento de Educação Continuada da SME, 184 concordaram com a sua participação na pesquisa, dos quais $97,82 \%$ pertenciam ao sexo feminino, e a idade média foi de 38,75 anos ( $d p=7,8$ anos). A formação mais apontada foi o Magistério, referida por $29,35 \%$ dos participantes, enquanto que o tempo que lecionavam variou de dois meses a 37 anos com média de 10,56 anos (dp=5,3 anos). Em relação à série que lecionavam, o maternal foi mencionado por $37,51 \%$ dos participantes, e $85,33 \%$ referiram que sua remuneração consistia em menos de três salários mínimos. Destaca-se que a maior parte dos participantes era de profissionais vinculados aos Centros Municipais de Educação Infantil (CEMEIS), que recebem crianças com idades entre zero e cinco anos.

A análise do conhecimento dos professores sobre o DM 1 evidenciou que a maior parte dos participantes, $58,15 \%$, foi capaz de definir corretamente a doença, relacionando-a a uma doença crônica caracterizada pela falta de insulina no organismo; porém, $64,67 \%$ professores acreditavam que o DM1 ocorria em pessoas de todas as faixas etárias.

A atividade física e a dieta específica foram apontadas por $33,70 \%$ dos participantes como as únicas formas de tratamento para o controle glicêmico. No que se refere à identificação dos possíveis sintomas que a criança pode apresentar durante as crises de hiperglicemia e hipoglicemia, evidenciou-se que, respectivamente, $27,72 \%$ e $22,83 \%$ dos participantes desconheciam a especificidade da sintomatologia de cada quadro, havendo necessidade da realização de exames e procura por ajuda profissional especializada na ocorrência destes eventos.

Entre os professores participantes do estudo, apenas dois citaram possuir alunos com diagnóstico médico de DM, e somente um respondeu às questões relacionadas ao conhecimento dos pais da criança e sobre a realização de reuniões periódicas com estes.

Em relação ao manejo da criança com crise hipoglicêmica, $42,40 \%$ dos professores afirmaram que não se deve oferecer açúcar em nenhuma hipótese e, sim, comunicar à mãe sobre o ocorrido, enquanto $28,26 \%$ não sabem como agir diante dessa situação, e 14 (7,60\%) não sabem ao certo o que é hipoglicemia.

Em se tratando do refeitório da escola, $71,20 \%$ dos professores afirmaram que os alimentos são padronizados para todas as crianças, não se considerando as necessidades individuais. Quando questionados sobre a existência de comunicação pela direção da escola, quanto à presença de alunos matriculados com diagnóstico médico de DM, apenas $15,21 \%$ dos professores responderam afirmativamente. 


\section{DISCUSSÃO}

O DM faz parte de um grupo de doenças metabólicas que se caracterizam pela hiperglicemia resultante da deficiência na secreção de insulina, na ação da insulina e/ou ambas. ${ }^{9}$ Essa doença está classificada em quatro subgrupos: diabetes mellitus tipo 1 (DM1), diabetes mellitus tipo 2 (DM2), outros tipos de diabetes associados a certas condições ou síndromes e o diabetes gestacional; porém, a maioria dos casos de DM está incluída em duas grandes categorias etiopatogênicas, ou seja, tipo 1 e tipo $2 .^{7-9}$

O DM1 ocorre em pessoas jovens, em decorrência da deficiência do hormônio, normalmente provocada pela destruição autoimune das células das ilhotas produtoras de insulina do pâncreas, levando à necessidade de injeções diárias de insulina para a manutenção das condições de sobrevivência. ${ }^{13}$

Os resultados deste estudo evidenciaram que a maioria dos professores denotou conhecimento sobre a definição de DM1, caracterizando-a como a ausência de insulina no organismo; entretanto, não conseguiram relacionar o DM1 como sendo mais frequente em pessoas jovens, normalmente com menos de 35 anos. ${ }^{14}$ Este dado leva a crer que existe um conhecimento de senso comum que circula sobre o significado da doença, fazendo com que as pessoas detenham algum saber, no entanto, muitas vezes são informações incompletas, equivocadas ou infundadas cientificamente.

Dentre as estratégias para a promoção da saúde e para a melhoria da qualidade de vida, o conhecimento constitui uma das mais poderosas ferramentas, pois capacita os cidadãos para o autocuidado e para a tomada de decisão sobre o que entende ser melhor para si próprio. Assim, os professores, como educadores e facilitadores da construção do conhecimento, necessitam também possuir conhecimento sobre a saúde do escolar.

Em relação ao tratamento do DM1, neste estudo, os professores apontaram a atividade física e a dieta específica como as únicas preditoras responsáveis pelo controle glicêmico. O tratamento para o DM1 tem por objetivo inicial a regulação dos níveis glicêmicos do indivíduo, sendo pautado na aplicação subcutânea de insulina, na dieta e na realização de exercícios físicos. Assim, a incorporação de atividades físicas e a adoção de uma dieta apropriada, associadas ao tratamento medicamentoso, são necessárias para o controle da hiperglicemia. ${ }^{8}$

Em um estudo realizado com professores de Educação Física, no município de Fortaleza, no Estado do Ceará, evidenciou-se déficit de conhecimento desses profissionais no manejo com pessoas com DM, salientando-se a necessidade de se implementar um programa de capacitação. As autoras enfatizam que todos os tipos de atividade física podem ser realizados por pessoas com DM, resultando em benefícios psíquicos e na atuação nos fatores de risco para doença macrovascular, dislipoproteinemia, hipertensão, obesidade e melhora do desempenho cardiovascular. ${ }^{17}$

Apesar da diversidade terapêutica atual, as manifestações clínicas da doença são comuns, e o tratamento do DM muitas vezes é considerado difícil e traumático para a criança, já que sua eficácia encontra-se ligada à disciplina e às mudanças nos hábitos de vida, havendo necessidade de esforços conjuntos entre a família, a criança e a escola ${ }^{1,5,18-19}$ Pesquisas mostram as dificuldades vivenciadas pela família, no contexto escolar, diante da insegurança quanto ao preparo dos educadores em lidar com as suas crianças. Reconhece-se que um ambiente seguro é aquele que dispõe de pessoas treinadas para medir a glicemia e reconhecer e tratar um episódio de hipoglicemia. ${ }^{20}$

No entanto, o reconhecimento dos sintomas apresentados pela criança, durante as crises de hiperglicemia e hipoglicemia, possivelmente não seria feito pela metade dos participantes deste estudo, uma vez que estes manifestaram desconhecimento sobre os sintomas apresentados em tais situações.

No contexto em que se insere a criança com DM, é fundamental que os cuidadores, sejam familiares ou professores, conheçam quais são os sintomas característicos dos quadros de hipoglicemia e de hiperglicemia. A hiperglicemia consiste na elevação da taxa de glicose no sangue, sendo caracterizada principalmente por sede intensa e aumento do volume urinário, podendo-se ainda citar a presença de náuseas e vômitos, sonolência e dor abdominal; já a hipoglicemia, trata-se de uma diminuição na taxa de glicose no sangue, caracteriza-se por suores, tremores, palidez, confusão mental, entre outros. ${ }^{5}$ Assim, ressalta-se que tanto a hiperglicemia como a hipoglicemia apresentam sinais e sintomas característicos que, se identificados a tempo, podem ser prontamente resolvidos.

O tempo de convivência entre a criança e o professor na escola é grande, visto que a criança passa parte de seu dia na instituição de ensino. Diante disso, é fácil para o professor observar mudanças em seu comportamento e em sua aparência física, por isso esses profissionais devem 
estar capacitados para reconhecer precocemente os sinais e sintomas adversos e tomar a decisão correta sobre o que fazer. Isso contribui para uma adequada intervenção e protege a criança de complicações agudas e crônicas.

Estudo demonstra que crianças de seis a 14 anos, portadoras de DM1, esperam que professores e colegas aprofundem seus conhecimentos sobre a doença. ${ }^{1}$ Este estudo enfatiza também que um conhecimento maior acerca da doença contribuiria positivamente para a melhor aceitação e continuidade da terapêutica, além de evitar consequências futuras para a criança diabética. ${ }^{21}$ Essa situação denota a falta de preparo dos educadores no que diz respeito à saúde do escolar, principalmente na temática sobre o DM.

Entende-se que é também uma responsabilidade da escola promover e contribuir para o bem-estar dos alunos, primando-se pela disponibilização das condições favoráveis ao processo ensinoaprendizagem. Importante questão a ser mencionada é a necessidade de um bom processo de comunicação entre escola e família, no sentido de informar sobre a real situação da criança, seu diagnóstico, suas necessidades, particularidades e possibilidades. Diante das informações, a instituição de ensino e seus profissionais deverão se preparar para a adequada abordagem da criança.

Foram reunidos em um estudo de revisão 40 artigos publicados, no período de 2000 a 2007, sobre os aspectos importantes para o cuidado da criança com DM no contexto escolar, destacandose a necessidade de oferecer à escola informações sobre a doença; elaboração de um plano de cuidado, contendo quem realizará o atendimento, o local onde ficará o material e as instruções sobre como poderá ser feito o tratamento; determinar e capacitar uma pessoa responsável pela administração do medicamento; comunicar aos pais quando houver viagens ou festas para que tomem as providências necessárias, orientar a criança sobre as escolhas alimentares mais adequadas nas festas e lanches escolares e instruir sobre a contagem de carbo-hidratos para calcular doses de insulina nos lanches e nas refeições, se necessário. ${ }^{20}$

Investigações sobre o preparo das escolas para ajudar a criança diabética demonstram que estas não se encontram devidamente preparadas e em condições para oferecer o cuidado de que a criança possa necessitar. ${ }^{22-23}$ Corrobora essas constatações uma revisão integrativa da literatura que analisou pesquisas sobre o atendimento e as intervenções da escola ao aluno com DM1, concluindo que são necessárias melhorias na comunicação entre escola e a família, na educação do pessoal, no cardápio alimentar e, ainda, na importância de disponibilizar um enfermeiro, tanto para o cuidado do aluno como para promover a educação dos funcionários da escola que muitas vezes prestam cuidados a estes alunos. ${ }^{24}$

Estudo realizado na Espanha procurou compreender o cotidiano das crianças com DM1, focalizando o período em que se encontram na escola. Professores foram questionados sobre o manejo do diabetes, evidenciando-se que o grau de conhecimento destes profissionais foi avaliado em três (média), numa escala de um a sete, e que eles se sentem inseguros, quando há uma criança com DM na escola, porque não têm instruções para agir, quando uma complicação ocorre..$^{25}$

Percebe-se que a atividade de educação em DM para os profissionais das escolas infantis é uma ferramenta primordial para o acompanhamento, cuidado e apoio à criança em suas necessidades; ademais, proporciona o sentimento de segurança e tranquilidade aos pais, durante o período em que a criança se encontra na escola. Sendo assim, há que se investir em atividades de educação em DM, pois, quanto mais os professores que acompanham a criança durante grande parte do seu dia conhecerem sobre o DM, mais fácil será manter sob controle a doença e o convívio da criança na escola. ${ }^{17}$

\section{CONCLUSÃO}

A realização deste estudo contribuiu para enfatizar a necessidade de capacitar os professores da educação infantil na temática DM, a fim de possibilitar a abordagem da criança com DM, no contexto escolar, com mais conhecimento, segurança e adequação.

Os resultados evidenciaram que, apesar de os professores denotarem conhecimento sobre o que é DM, os mesmos demonstraram desconhecer os sinais e sintomas da hipo e da hiperglicemia; as abordagens terapêuticas e as principais condutas a serem adotadas diante de intercorrências glicêmicas.

A falta de capacitação do professor para lidar com a criança com DM, enquanto esta se encontra sob sua responsabilidade no ambiente escolar, gera dificuldades e insegurança para este profissional que não se sente apto a intervir e a proporcionar o cuidado adequado. Por sua vez, este despreparo da escola em manejar essa situação traz para a família intranquilidade e apreensão, por estar sempre imaginando o que pode suceder, caso 
seu filho venha a necessitar de cuidados durante o período em que está escola.

Acredita-se que as instituições de ensino devem apresentar recursos físicos, materiais e humanos compatíveis às necessidades de aprendizagem das crianças; nisto está implícita a formação de seus professores que, além de dominarem o conhecimento específico de sua área de atuação, devem apresentar razoável bagagem de conhecimentos gerais, especialmente no que concerne à saúde do escolar.

É importante salientar que a pesquisa teve como principal limitação, o fato de restringir-se a professores da educação infantil, não abarcando professores de outros níveis que eventualmente convivem com a problemática ora suscitada.

Apesar de a literatura já ter explorado de modo pertinente o tema abordado, os resultados deste estudo ainda apontam para a necessidade de políticas públicas e do fortalecimento de ações intersetoriais que promovam a melhor compreensão da realidade que permeia a saúde e a educação das crianças, focalizando o preparo e a capacitação dos professores que assumem a responsabilidade pela formação dos cidadãos.

\section{REFERÊNCIAS}

1. Nunes MDR, Dupas G. Entregando-se à vivência da doença com o filho: a experiência da mãe da criança/ adolescente diabético. Texto Contexto Enferm. 2004 Jan-Mar; 13(1):83-91.

2. Marcon SS, Radovanovic CAT, Waidman MAP, Oliveira MLF, Sales CA. Vivências e reflexões de um grupo de estudos. Texto Contexto Enferm. 2005; 14(Esp):116-24.

3. Pilger C, Abreu IS. Diabetes mellitus na infância: repercussões no cotidiano da criança e de sua família. Cogitare Enferm. 2007 Out-Dez; 12(4):494-501.

4. Moreira OL, Dupas G. Vivendo com o diabetes: a experiência contada pela criança. Rev Latino-am Enfermagem. 2006 Jan-Fev; 14(1):25-32.

5. Zanetti ML, Mendes IAC. Análise das dificuldades relacionadas às atividades diárias de crianças e adolescente com diabetes mellitus tipo 1: depoimento de mães. Rev Latino-am Enfermagem. 2001 NovDez; 9(6):25-30.

6. Rodrigues TMB, Silva IN. Estatura final de pacientes com diabetes mellitus do tipo 1. Arq Bras Endocrinol Metab. $2001 \mathrm{Fev}$; 45(1):47-54.

7. Beaglehole R. Informes do diabetes no mundo. Diabetes care. 2004 Mar; 8(3):162.

8. American Diabetes Association. Prevention of type 1 diabetes. Diabetes care. 2004; 27(Suppl 1):133-6.
9. American Diabetes Association. Diagnosis and classification of diabetes mellitus. Diabetes Care. 2007; 2(Suppl 1):42-7.

10. Trevisan E, Lenardt MH, Jouclas UMG. O cuidado à criança diabética na teia da vida. Cogitare Enferm. 1998 Jul-Dez; 3(2):11-4.

11. Zanetti ML, Mendes IAC, Ribeiro KP. O desafio para o controle domiciliar em crianças e adolescentes diabéticas tipo 1. Rev Latino-am Enfermagem. 2001 Jul; 9(4):32-6.

12. Stacciarini TSG, Haas VJ, Pace AE. Fatores associados à autoaplicação de insulina nos usuários com diabetes mellitus acompanhados pela Estratégia Saúde da Família. Cad. Saúde Publica. 2008 Jun; 24(6):1314-22.

13. Rudolph AM, Kamel RK. Rudolph: princípios de pediatria. São Paulo (SP): Roca, 1997.

14. Sociedade Brasileira de Diabetes. Tudo sobre diabetes; 2010 [acesso 2010 Jan12]. Disponível em: http://www.diabetes.org.br/tudo-sobre-diabetes

15. Sampaio FAA, Melo RP, Rolim ILTP, Siqueira RC, Ximenes LB, Lopes MVO. Avaliação do comportamento de promoção da saúde em portadores de diabetes mellitus. Acta Paul Enferm. 2008; 21(1):84-8.

16. Novato TS, Grossi SAA, Kimura M. Qualidade de vida e auto-estima de adolescentes com diabetes mellitus. Acta Paul Enferm. 2008; 21(4):562-7.

17. Monteiro LZ, Spinato IL, Pinheiro MHNP, Silva $\mathrm{CAB}$ da, Montenegro Júnior RM. Exercício físico em crianças com diabetes mellitus tipo 1: conhecimento do profissional de educação física. Rev Bras Ci e Mov. [online]. 2009[acesso 2010 Jan 14]; 17(2):1-23. Disponível em: http:/ / portalrevistas.ucb.br/index. php/RBCM/article/viewFile/897/940E

18. Góes APP, Vieira MRR, Libertore Junior RDL. Diabetes Mellitus tipo 1 no contexto familiar e social. Rev Paul Pediatria. 2007 Jun; 25(2):24-8.

19. Francioni FF, Silvia DGV. O processo de viver saudável de pessoas com diabetes mellitus através de um grupo de convivência. Texto Contexto Enferm. 2007 Jan-Mar; 16(1):105-11.

20. Leite SAO, Zanim LM, Granzotto PCD, Heupa S, Lamounier RN. Pontos básicos de um programa de educação ao paciente com diabetes melito tipo 1. Arq Bras Endocrinol Metab [online]. 2008 Mar [acesso 2010 Jan 13]; 52(2):233-42. Disponível em: http:// www.scielo.br/pdf/abem/v52n2/07.pdf

21. Nimsgern AMPH, Camponeschi, JMS. Implementing a new diabetes resource for Wisconsin Schools and families. Centers for Disease Control and Prevention [online]. 2005 Nov [acesso 2010 Jan 11]; 2(Esp):1-11. Disponível em: www.cdc.gov/pcd/issues/2005/ nov/05_0081.htm

22. Tahirovic H, Toromanovic A. How much can school staff help children with diabetes type 1 in school? Med Arh. [online]. 2006 [acesso 2010 Jan 
14]; 60(4):222-4. Disponível em: http://www.ncbi. nlm.nih.gov/pubmed/16761513

23. Tahirovic H, Toromanovic A. How far are physical education teachers from elementary school prepared to help pupils with diabetes while they are at school? Minerva Pediatr [online]. 2007 Dec [acesso 2010 Jan 14]; 59(6):767-73. Disponível em: http:/ / www.ncbi. nlm.nih.gov/pubmed/17978786

24. Tolbert R. Managing type 1 diabetes at school: an integrative review. J Sch Nurs [online]. $2009 \mathrm{Feb}$ [acesso 2010 Jan 11]; 25(1):55-61. http:/ / www.ncbi. nlm.nih.gov/pubmed/19197019

25. Manchón MG, Carrasco JG, Fernández JR, Fernández TD, Frías EG. Necesidades del niño escolarizado con diabetes mellitus: visión de padres y profesores. An Pediatr (Barc). 2009 [acesso 2010 Jan 13]; 70(1):45-52. Disponível em: http://www.elsevier.es/revistas/ ctl_servlet?_f=7064\&ip=189.41.88.52\&articuloid $=1$ $3131547 \&$ revistaid $=37$

E-mail: assisimoes@yahoo.com.br 